\title{
The effects of golf training in patients with stroke: a pilot study
}

\author{
Tobias Schachten and Petra Jansen
}

Institute of sport science, University of Regensburg, Germany

ABSTRACT

Background: Stroke is the most common neurological disease and the primary cause of lifelong disability in industrialized countries. Because of this it is important to investigate any kind of successful therapy.

Methods: From the 24 recruited stroke patients who were between 23 and 72 years old, 14 patients were separated either in a golf training group (EG), or a social communication meeting (CG). Both groups met for one hour sessions, twice a week, for ten weeks. All participants completed assessment tests before and after the experimental period: cognitive tests measuring attention (Go/No-Go task), visual-spatial memory (Block-Tapping test) and mental rotation performance (MRT); a balance test (Berg Balance Scale), and an emotional well-being test (CES-D-Scale).

Results: The results show that both groups improved in the CES Scale, the block-tapping test and the balance test. In addition, stroke patients who received a golf training showed a significant improvement in the MRT comparing to the control group (CG).

Conclusion: It is indicated that golf training can improve visual imagery ability in stroke patients, even late after stroke.

Key words stroke, golf exercises, rehabilitation, cognition, motor skills, emotions, visual-spatial cognition

\section{Introduction}

The main goal of this study is to investigate the effectiveness of a coordinative specific golf training for patients late after stroke. Because stroke is the most common neurological disease, with approximately one million first-time stroke incidents in Europe each year (Truelsen et al., 2006), and the primary cause of lifelong disability in industrialized countries (Johnston et al., 2009), it is important to investigate any kind of successful therapy. There are several different forms of stroke, which have different causes and effects. The most frequent form is the ischemic stroke. Here, blood supply to a specific part of the brain is reduced; accordingly this area of the brain no longer functions properly. Ischemic stroke occurs mainly in people over 65 years old, however, $25 \%$ of patients are younger than 60 years and $15 \%$ are younger than 45 years. Because of the modern lifestyle, risk factors (physical inactivity, increasing diabetes, and obesity disorders) for suffering a stroke have

Correspondence should be addressed to: Tobias Schachten, Institute of sport science, University of Regensburg, Universitätsstr. 31, 93053 Regensburg, Germany. Phone: +49-941-943-2445. Email: tobias.schachten@ur.de. Received 18 Aug 2014; revision requested 6 Oct 2014; revised version received 14 Oct 2014; accepted 21 Oct 2014. First published online 8 January 2015 increased. Depending on which area of the brain is affected, stroke patients often experience a variety of neuropsychological and motor deficits that can reduce their cognitive, emotional, and motor performance. These disabilities have been a matter of research and it has been shown that cognitive processes such as perception, memory, attention, language, and executive function are typically impaired (Chen et al., 2013). There are also deficits in motor performance such as paresis, loss of balance or mobility. Consequently, there is a change in quality of life, which often leads to mood disorders such as depression.

\section{Physical rehabilitation after stroke}

Beside the well-known health-promoting effects of physical activity in healthy people (Duncan et al., 1998; Shepherd, 2001) exercise therapy is an integral part in the post-stroke rehabilitation process with a favorable effect on activities of daily life in patients with stroke (Kwakkel et al., 2004). Motor functions in stroke patients can be improved by special physical training such as circuit training (van de Port et al., 2012), cycle ergometer intervention (Potempa et al., 1995), or bilateral movement 
training (Cauraugh and Summers, 2005). A metaanalysis showed that task-oriented circuit training compared to usual therapeutic exercise enhances gait speed, walking distance, and stair climbing exercise performance (Wevers et al., 2009). Duncan et al. (2003) investigated the influence of a 12week exercise therapy compared to a traditional rehabilitation program in improving balance, agility, strength, endurance, and functionality of the lower extremities. Compared to the CG the experimental group (EG) showed significant progress in balance, endurance, and agility.

In summary it can be assumed that augmented exercise enhances motor skills after stroke. Furthermore, there is some evidence that exercise also increases cognitive performance (Colcombe and Kramer, 2003; Angevaren et al., 2008). Cumming et al. (2012) consider in a review of twelve studies, the influence of physical activity on cognitive function in stroke patients. They showed that increased physical activity primarily benefits the working memory, attentional skills, visual-spatial cognition, and executive functions (Pyöriä et al., 2007; das Nair and Lincoln, 2008).

Pyöriä et al. (2007) compared the effects of a 12 month activating therapy to a traditional therapy group and demonstrated a significant improvement in the activating therapy group in terms of memory, visual-spatial, and visual inattention while no improvements were perceived in the traditional therapy group. According to the review of Hillman et al. (2008) there is a growing number of intervention studies which have examined whether fitness training has a positive effect on different aspects of perception and cognition. Based on the growing amount of evidence it can be assumed that there is a relationship between increased physical activity and cognitive performance. However, there are few studies that investigate the type of movements which are optimal for cognitive impact.

Marzolini et al. (2013) showed that a combination of strength and endurance training after a stroke leads to general cognitive improvements, especially in the areas of attention and executive functions. Quaney et al. (2009) examined the influence of eight-week aerobic exercise training on cognitive and motor functions compared to a stretching intervention. There were significant improvements in cognitive processing speed for the aerobic group compared to the stretching group.

It is assumed that emotional well-being and cognitive functioning may be related to each other. In addition, it is indicated that physical activity can influence the psychological well-being (Roth, 1989). Most studies on the topic were conducted with older people as participants (Arent et al., 2000; Strawbridge et al., 2002).
Unfortunately, there are not many studies which investigate the effects of exercise on emotional well-being in stroke patients. Lai et al. (2006) demonstrated that exercise may help decrease poststroke depressive symptoms. In this study the treatment included flexibility, strength, balance, and endurance exercises. Penninx et al. (2001) have also shown that aerobic exercise can lower depressive symptoms in stroke patients.

To summarize, there are currently many studies demonstrating that exercise-based therapy can enhance the motor, emotional, and cognitive recovery after stroke. But there are fewer studies regarding the effects of specific exercise treatments, such as coordinative exercises.

\section{Influence of coordinative training and golf on cognitive, emotional and physical well-being}

Coordination is the interaction of sensory organs, peripheral and central nervous system, and muscles. Coordinative training focuses on an improvement of balance, eye-hand coordination, agility, and accuracy. The everyday life and a lot of different sports demand a high level of coordination skills. The effect of a coordinative training, karate in this study, has already shown for emotional well-being in older healthy adults (Jansen and Dahmen-Zimmer, 2012). Studies with stroke patients were quite rare: Boiko et al. (2008) showed that specific coordinative exercises which include "brain gymnastics" improve cognitive functions, mood, and quality of life in post-stroke patients. In this study 82 patients were randomly allocated to two groups. The EG participated in brain gymnastic exercises; the CG joined a standard rehabilitative treatment. Caraugh and Summers (2005) also confirmed a positive relationship between coordinative exercises and cognition after stroke. They assumed that bilateral movements have potential to expedite the progress of chronic stroke recovery. They concluded that executing bilateral movements post-stroke may facilitate cortical neural plasticity (Cauraugh and Summers, 2005). Furthermore, golf as a coordinative sport has been investigated in the poststroke research. Shatil et al. (2005) have shown that golf as a rehabilitation measure leads to better functional balance and quality of life than a conventional physiotherapy.

\section{GOAL OF THIS STUDY}

The main goal of this study was to investigate whether there is a positive influence of coordinative golf exercises in stroke patients late after stroke regarding their cognitive, emotional, and physical performance. These specific golf exercises were chosen because they include a high amount 
Table 1. Age, time of stroke, and number of sessions for each group

\begin{tabular}{|c|c|c|c|}
\hline & AGE（YEARS） & $\begin{array}{l}\text { TIME OF STROKE } \\
\text { (YEARS AFTER STROKE) }\end{array}$ & $\begin{array}{l}\text { NUMBER OF } \\
\text { SESSIONS }\end{array}$ \\
\hline Golf training group & $M=55.14(\mathrm{SD}=17.64)$ & $M=3.43(\mathrm{SD}=1.81)$ & $M=18.14(\mathrm{SD}=1.57)$ \\
\hline Social communication group & $M=53.14(\mathrm{SD}=13.54)$ & $M=3.86(\mathrm{SD}=1.67)$ & $M=19.14(\mathrm{SD}=0.37)$ \\
\hline
\end{tabular}

of coordinative exercises, which are supposed to relate to cognition (Caraugh and Summers, 2005). Furthermore, more evidence came from a neuroscientific study with experience golfer who show a higher amount of grey matter in a specific brain area which relates to visual-spatial performance. To determine the effects of golf, we included an EG which received a specific coordinative golf training and a CG which attended a communication meeting. We hypothesized that the EG would show a greater improvement in balance ability, well-being, visual-spatial memory span, and MRT compared to the CG after the ten week treatment.

\section{Method}

\section{Participants}

In total 24 patients were recruited by advertisements in the local radio, newspapers, and the local neurological rehabilitation clinic. Because they differed in age and time since the stroke incident, they were matched in pairs by age and date of the stroke. This resulted in seven pairs with 14 patients. After this one patient of this pair was assigned to one of the two groups, either the golf training or the social communication group. The patients of each group did not differ according to age $\mathrm{t}(12)=0.238$, n.s., time of the stroke, $\mathrm{t}(12)=-1.635$, n.s., or the time they participated in the training sessions, $\mathrm{t}(12)=-1.635$, n.s., (see Table 1).

None of the patients had experience in playing golf before participating in this study. Independent of this golf intervention all patients took part in common treatments such as physiotherapy and occupational therapy for one hour twice a week.

The ethical review committee was informed.

\section{Material}

TESTS

All participants completed assessment tests before and after the intervention: cognitive tests measuring inhibition (Go/No-Go task), visual-spatial memory (Block-Tapping task) and MRT; a balance test (Berg Balance Scale); and an emotional well-being test (CES-D-Scale). The tests are described as follows.

Go/No-Go task (Nosek and Banaji, 2001)

The Go/No-Go task is a computerized method that validates inhibition as the ability to suppress non-adequate responses to irrelevant stimuli. Participants must respond to only one of the two possible stimuli which are randomly presented. While one stimulus requires a response the other stimulus does not. The test consists of a single run of 100 stimuli presented on a computer screen, 70 stimuli require a response and 30 do not. Dependent variables are the reaction time and the number of incorrect responses. The objectiveness of the Go/No-Go task is very high, because it is a standardized instruction presented on the screen.

Block-Tapping task (BTT) (Corsi, 1972)

The BTT is a test used to assess visual-spatial short term working memory.

The test instrument consists of a base plate with nine wooden blocks attached to the surface. The experimenter taps a series of blocks on the board, moving to a new block every second. Then, the participant must tap in the same order. The test consists of eight items, each with three trials. For each correctly repeated sequence, the participant receives one point, so that a maximum of 24 points can be achieved. The dependent variable is the number of points earned, which measures the visual-spatial memory.

Mental Rotation Test (MRT) (Peters et al., 1995)

Mental rotation ability was measured using a psychometric MRT (paper and pencil test). The test procedure lasted about $15 \mathrm{~min}$ per participant. The participants were presented with sheets of paper that had a standard object on the left and four comparison objects on the right side (see Figure 1). Two of the comparison items were the same and two were mirror reversed. Participants had to decide which of the two comparison items were the same as the standard item. There were a total of 24 tasks presented in two blocks with 12 tasks each. The participants were given three minutes to solve each block. One point was given only if both correct sample stimuli of a cube figure were identified correctly. Participants could achieve a maximum 

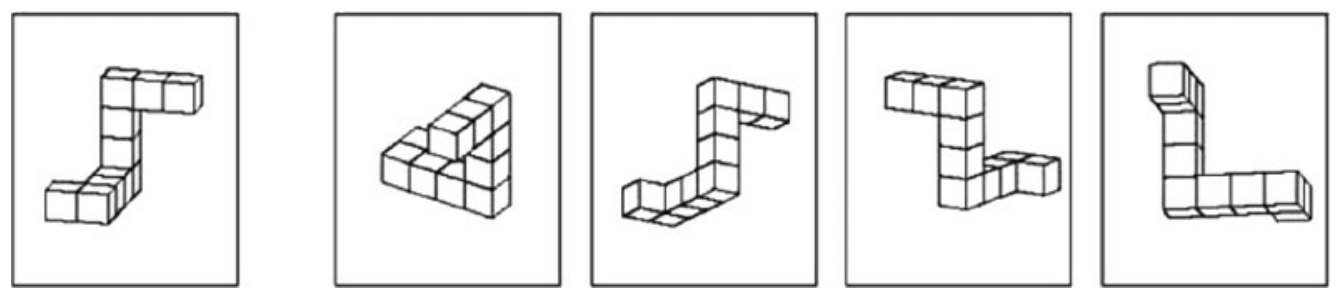

Figure 1. One task in the Mental Rotation Test of Peters et al. (1995).

of 24 points. The dependent variable was the total score of correct items received in both test blocks.

Berg Balance Scale (BBS) (Berg et al., 1989)

The BBS is a 14 item scale designed to measure balance of the older adults and is especially suitable for patients with neurological disorders. There are 14 items that are scored on a range from 0 to 4 . A score of 0 represents the inability to accomplish the task while a score of 4 is given if the participant completes the task correctly. A maximum score is 56 possible points. Scores of 0-20 demonstrate balance impairment, 21-40 represent suitable balance, and 41-56 show good balance. The items include simple mobility tasks (ex: transfers, standing unsupported, sit-to-stand) and more difficult tasks (ex: tandem position, turning $360^{\circ}$, single-leg position). The test has a reliability and objectivity of $r=0.99$ and $r=$ 0.98 .

CES-D-Scale (Hautzinger and Bailer, 1993)

The CES-D-scale, here a short form, is a common screening tool for self-assessment of depressive symptoms. The questions refer to the last week and have to be evaluated with a four-point response option $(0=$ rarely or none of the time; $3=$ more or all of the time). Total scores are calculated by adding response values for the 15 individual items. The CES-D total score can range from 060 points. Higher scores indicate strong depressive symptoms. A score of 17 indicates the existence of a serious depressive disorder.

\section{Training sessions}

\section{GOLF TRAINING}

During the 10 week period the patients of the EG received golf training twice a week for $1 \mathrm{~h}$, which resulted in 20 sessions. The training sessions provided instruction for specific golf exercises thought to enhance the cognitive and motor performance of stroke patients.

Patients performed specific coordinative exercises with a golf club and a ball on a putting green in the golf room of a neurological clinic. The aim was to introduce golf, both establishing the patient's first contact with the sport and teaching them basic techniques under simplified conditions.
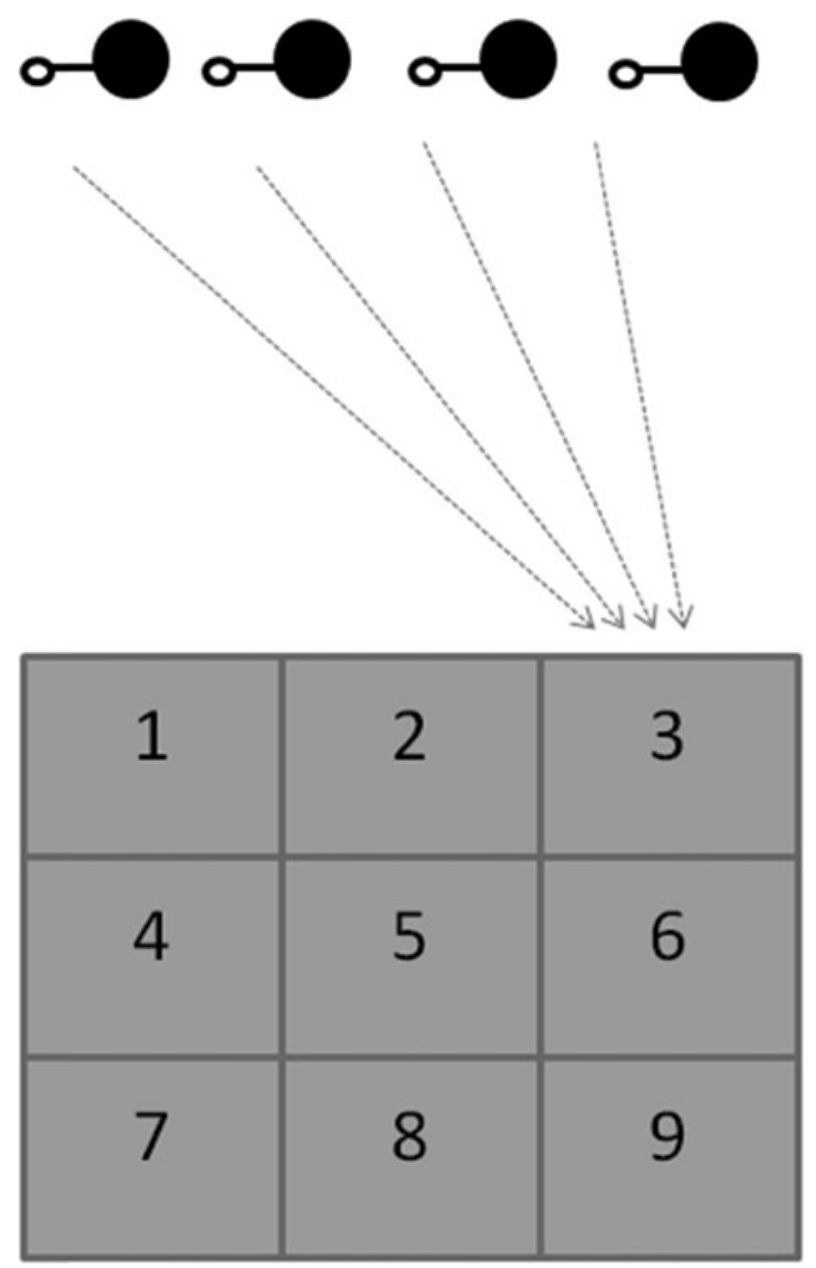

Figure 2. An example of a specific golf task that has been performed during the intervention.

Priority was given to the development of eye-hand coordination and balance ability. To achieve this, most of the exercises included a combination of typical golf movements and additional cognitive tasks. For example: Participants were presented with several numbered areas in which the ball must be played with a golf club (see Figure 2). They were all asked at the same time: In which month does spring start? The participants had to think about the correct answer before trying to play the ball in the corresponding area (in this case it was area number 3). Each exercise included a 
combination of performing a cognitively demanding task and a coordinative activity. All patients were able to perform the exercises safely. The training was adapted to the abilities of each patient based on their individual impairments. If a patient was not able to use both hands due to hemiparesis, a so called broomstick putter or belly stick putter was given to him, which offered him an additional stability. Another patient with visual impairment used a colored golf ball instead of an usual white ball.

\section{Social communication group}

Patients of the CG engaged in a social communication meeting including group discussion, reading, and common game activities. In the beginning, we focused on reading and talking. For this, the participants had to take turns reading stories out loud to each other. Various stories were used, such as topics of the daily newspaper or crime stories. After the text was read, the investigator directed questions to the participants to initiate a discussion. The CG also played various parlor games such as the card game Memory (also known as Pairs). Games were used to include a cognitive component. All patients were included in all games equally. Patients attended the control treatment for ten weeks, twice a week, for one hour, which resulted in a maximum of 20 sessions.

\section{PROCEDURE}

Each group participated in either golf training (EG) or a social communication meeting (CG). Both groups met for one hour sessions, twice a week, for ten weeks. The tests were completed one week before the intervention started again one week after the intervention finished.

\section{DESIGN}

This study has a pre-post design with two different groups, an EG, which received the golf training and a CG, a social communication group. Dependent measurements were the pre- and posttest performance described above.

\section{Statistical analysis}

Due to the small sample size and the fact that not in every measurement normal distribution was reached, we used non-parametric analysis. First of all, we used the Mann-Whitney U-Test to investigate, if there is a group difference in the pretest measurements. Second, we analyzed if patients improved their performance from the first test to the second test in each dependent measurement using the Wilcoxon-Test., overall and for each group. In the dependent measurements in which an improvement was shown we calculated the difference values for each measurement, and used the Mann-Whitney-Test to determine if group (golf group vs. social communication group) had a significant influence on final performance.

\section{Results}

\section{Pre-test differences}

The Mann-Whitney U-Test showed only a significant difference in the pre-test for the BBS between groups, with a significant better performance of the golf training group, $\mathrm{U}=$ $6, \mathrm{z}=-2.40, p<0.05$. None of the pretest measurements showed a significant correlation between the years since the stroke appeared and the pre-test measurements.

\section{Overall pre- and post-test outcome}

The results show a significant improvement in the post-test, independent of the factor "group", for the dependent measurements: mental rotation, $z=$ $-2.11, p<0.05$; depression, $z=-2.21, p<0.05$; Go/No-Go task (only analysis of error rate), $z=$ $-1.99, p<0.05$; BBS, $z=-2.36, p<0.05$; and an almost significant difference for the block-tapping test, $z=-1.93, p=0.054$. All means and standard deviations are presented in Table 2.

\section{Pre-and Post-test outcome within each group}

For the golf training group there was a significant improvement in the post-test compared to the pre-test for the following measurements: mental rotation, $z=-2.38, p<0.05$; depression, $z=$ $-2.21, p<0.05$; and or the block-tapping test, $z$ $=-2.23, p<0.05$. For the social communication group none of the post-test showed a significant improvement compared to the pre-test.

\section{Group difference}

The Mann-Whitney test only showed an influence of the factor "group" for the difference score in the MRT, $\mathrm{U}=6, z=-2.42, p<0.05$. The golf training group $(M=3.51, S D=1.9)$ improved more from the pre- to the post-test than the social communication group $(\mathrm{M}=.28 ; \mathrm{SD}=2.42)$. The effect size was high, $d=1.49$. In all other difference scores there was no influence of the factor "group" (all $p>0.01$ ). All effect sizes $(d)$ in the difference score between groups of each dependent measurements are given in Table 2. 




\section{Discussion}

Concerning our hypotheses, that the EG shows a greater improvement in balance ability, well-being, visual-spatial memory span, and MRT compared to the CG after the ten week treatment, we only could show a greater improvement in the MRT. Both groups (EG and CG) improved in emotional well-being, balance, inhibition, and visual-spatial short term working memory. Investigating this improvement in more detail it was shown, that social communication group did not improve significantly but the effect sizes in Table 2, especially for the Go/No-Go test show, that this result might be due to the small sample size. With a larger sample size, a significant improvement at least for attention and inhibition measurement should be found also for the social communication group. This is in line with literature, which shows that social interaction plays an important role in various domains of learning (Meltzoff et al., 2009).

For the Golf training group, the improvement for the MRT, the block-tapping test and the depression score revealed significance. These results compared to the overall significant improvements of both group is due to the small sample size in each group.

The significant improvement in MRT, the ability to imagine an object rotated from its original position, after golf exercises suggests that golf training can improve visual imagery ability in stroke patients. Furthermore, as mentioned in the introduction it is in line with a study of Jäncke et al. (2009) who showed that skilled golfers had higher grey matter volumes in the intraparietal sulcus, a brain area which is also activated during a MRT (Jordan et al., 2001). The result that golf, a coordinative sport, can have influence on MRT is comparable to other studies investigating motor training on MRT. As Wiedenbauer et al. (2007) have already presented, manual rotation training, such as hand rotations or eye-hand coordination tasks enhances MRT in adults. Wohlschlaeger and Wohlschlaeger (1998) have hypothesized that mental and motor rotations share the same neural processes which control both the imagined and the physical rotation. This supports studies which have examined the relationship between coordinative tasks, such as juggling, and visual imagery ability. The study of Draganski et al. (2004) helps to confirm this idea by demonstrating that changes in neurological processes occur due to coordinative tasks. Jansen et al. (2009) have specifically examined the influence of juggling on MRT and found that juggling training does improve MRT.

There is a lot of evidence to support a relationship between coordinative exercise and visual imagery performance, however there are 
no studies investigating those effects in stroke patients. We only found one study examining the effectiveness of golf training after stroke (Shatil et al., 2005). But the main emphasis has never been put on the mental rotation.

The improvement in MRT in this study might be due to the fact that the golf exercises included additional visual imagination tasks. Research has been conducted investigating the effects of dual-task conditions in stroke patients (Hyndman et al., 2006; Lord et al., 2006; Yang et al., 2007). The dual-task paradigm or cognitive-motor interference (CMI) conditions combine motor tasks and cognitive tasks in one exercise. The effects of those CMI conditions have been observed across all ages, especially following stroke (Dennis et al., 2009). The improvement in mental rotation is very important because one might expect transfers to other tasks. This ability is indispensable for everyday life: to reach for a cup of coffee, we have to know the start position of the hand and the target localization of the cup. It is well established that mental rotation ability is important for different cognitive aspects such as problem solving (Geary et al., 2000), mathematics (Hegarty and Kozhevnikov, 1999), and science (Peters et al., 1995).

The result of the positive effect of golf exercises on mental rotation is emphasized by the positive effect on the visual-spatial memory test. Even the improvement for the golf training group did not reach significance, the high effect size $(d=0.95)$ gives a hint that the non-significance might be only due to the small sample size. This result justifies larger size trials in further studies.

The present study is similar to that of Boiko et al. (2008); they confirmed positive effects of brain gymnastics on cognitive function, emotional well-being and quality of life in stroke patients. We recommend that future studies also use neuromuscular or coordinative tasks to further investigate more effective therapies for stroke rehabilitation.

Additionally, there is an increasing body of evidence that suggests that exercise after stroke has a number of benefits. This is the reason for official exercise program recommendations post-stroke. Neuromuscular exercises are also recommended, such as aerobic or strength training (Gordon, 2004). The present study confirms and adds to the findings that coordinative training is effective for stroke rehabilitation. Additionally, the use of golf training for rehabilitation of other neurological disorders should be investigated.

In addition to the positive results on MRT for the EG group, both groups improved in emotional well-being, balance, inhibition, and visual-spatial short term working memory. This suggests that any type of group activity may help to improve emotional and cognitive symptoms. This is an important result which should be considered further in rehabilitation programs. The CG was a social group which performed cognitive tasks, such as playing "pairs", for this, the improvement in some cognitive measurements seems to be possible.

None of the patients were in the acute phase, meaning that the stroke occurred on an average of 3.5 years earlier. This suggests that even after the critical period for stroke rehabilitation has passed, social and coordinative activity can enhance cognitive performance. One indication for the high value of both groups is the great compliance of the patients; each participant attended at least 18 of the 20 training sessions.

\section{Limitations}

The present study has shown that a specific golf training can enhance visual-spatial imagery in patients with late stroke. The current study is limited by the small number of participants, for this it can only be seen as a pilot study. The time intensive nature of the program only allowed patients from the region to be included. Patients were matched according to age and date of the stroke incident to meet high experimental criteria, but the small number of patients in each group might prevent applying this result to the general population. Furthermore, the length of the treatment should be increased in further studies and the sustainability of the effects should be investigated when golf training and social communication are eliminated.

\section{Conflict of interest}

None.

\section{Description of authors' roles}

Tobias Schachten designed the study, supervised the data collection and wrote the paper. Petra Jansen was responsible for the statistical design of the study and for carrying out the statistical analysis.

\section{Acknowledgments}

We are grateful to the patients who took part at the project. Furthermore, we thank Oliver Kern for helping during data acquisition and Maria Dotzler and Dr. Weber at the Neurological clinic of Regensburg and the Association "Zweites Leben $\mathrm{eV}$ ". We greatly appreciate their assistance. 


\section{References}

Angevaren, M., Aufdemkampe, G., Verhaar, H. J. J., Aleman, A. and Vanhees, L. (2008). Physical activity and enhanced fitness to improve cognitive function in older people without known cognitive impairment. Cochrane database of systematic reviews (Online), 3, CD005381.

Arent, S. M., Landers, D. M. and Etnier, J. L. (2000). The effects of exercise on mood in older adults: a meta-analytic review. Fournal of Aging and Physical Activity, 8, 407-430.

Berg, K., Wood-Dauphinee, S., Williams, J. I. and Gayton, D. (1989). Measuring balance in the elderly: preliminary development of an instrument. Physiotherapy Canada, 41, 304-310.

Boĭko, E. A., Kulishova, T. V., Shumakher, G. I. and Iusupkhodzhaev, R. V. (2008). The role of physical exercises in the improvement of cognitive functions in patients who survived stroke, in the early rehabilitative period. Voprosy Kurortologii, Fizioterapii, i Lechebnǒ Fizicheskoi Kultury, 6, 9-12.

Cauraugh, J. H. and Summers, J. J. (2005). Neural plasticity and bilateral movements: a rehabilitation approach for chronic stroke. Progress in Neurobiology, 75, 309-320.

Chen, C., Leys, D. and Esquenazi, A. (2013). The interaction between neuropsychological and motor deficits in patients after stroke. Neurology, 80 (Suppl. 2), S27-S34.

Colcombe, S. and Kramer, A. F. (2003). Fitness effects on the cognitive function of older adults: a meta-analytic study. Psychological Science, 14, 125-130.

Corsi, P. M. (1972). Human memory and the medial temporal region of the brain. Dissertation Abstracts International, 34, 819B.

Cumming, T. B., Tyedin, K., Churilov, L., Morris, M. E. and Bernhardt, J. (2012). The effect of physical activity on cognitive function after stroke: a systematic review. International Psychogeriatrics/IPA, 24, 557-567.

das Nair, R. and Lincoln, N. (2008). Effectiveness of memory rehabilitation after stroke. Stroke, 39, 516.

Dennis, A. et al. (2009). Fast walking under cognitive-motor interference conditions in chronic stroke. Brain Research, 1287, 104-110.

Draganski, B., Gaser, C., Busch, V., Schuierer, G., Bogdahn, U. and May, A. (2004). Neuroplasticity: changes in grey matter induced by training. Nature, 427, 311-312.

Duncan, P. et al. (1998). A randomized, controlled pilot study of a home-based exercise program for individuals with mild and moderate stroke. Stroke; a fournal of Cerebral Circulation, 29, 2055-2060.

Duncan, P. et al. (2003). Randomized clinical trial of therapeutic exercise in subacute stroke. Stroke; a fournal of Cerebral Circulation, 34, 2173-2180.

Geary, D. C., Saults, S. J., Liu, F. and Hoard, M. K. (2000). Sex differences in spatial cognition, computational fluency, and arithmetical reasoning. Fournal of Experimental Child Psychology, 77, 337-353.

Gordon, N. F. (2004). Physical activity and exercise recommendations for stroke survivors: an American heart association scientific statement from the council on clinical cardiology, subcommittee on exercise, cardiac rehabilitation, and prevention; the council on cardiovascular nursing; the council on nutrition, physical activity, and metabolism; and the stroke council. Circulation, 109, 2031-2041.

Hautzinger, M. and Bailer, M. (1993). Allgemeine Depressions Skala - ADS. Weinheim: Beltz.

Hegarty, M. and Kozhevnikov, M. (1999). Types of visual-spatial representations and mathematical problem solving. Fournal of Educational Psychology, 85, 479-500.

Hillman, C. H., Erickson, K. I. and Kramer, A. F. (2008). Be smart, exercise your heart: exercise effects on brain and cognition. Nature Reviews Neuroscience, 9, 58-65.

Hyndman, D., Ashburn, A., Yardley, L. and Stack, E. (2006). Interference between balance, gait and cognitive task performance among people with stroke living in the community. Disability and Rehabilitation, 28, 849-856.

Jäncke, L., Koeneke, S., Hoppe, A., Rominger, C. and Hänggi, J. (2009). The architecture of the golfer's brain. PloS one, 4, e4785.

Jansen, P. and Dahmen-Zimmer, K. (2012). Effects of cognitive, motor, and karate training on cognitive functioning and emotional well-being of elderly people. Frontiers in Psychology, 3, 40.

Jansen, P., Titze, C. and Heil, M. (2009). The influence of juggling on mental rotation performance. International Fournal of Sport Psychology, 40, 351-359.

Johnston, S. C., Mendis, S. and Mathers, C. D. (2009). Global variation in stroke burden and mortality: estimates from monitoring, surveillance, and modelling. The Lancet Neurology, 8, 345-354.

Jordan, K., Heinze, H. J., Lutz, K., Kanowski, M. and Jäncke, L. (2001). Cortical activations during the mental rotation of different visual objects. NeuroImage, 13, 143-152.

Kwakkel, G. et al. (2004). Effects of augmented exercise therapy time after stroke: a meta-analysis. Stroke; a fournal of Cerebral Circulation, 35, 2529-2539.

Lai, S.-M., Studenski, S., Richards, L., Perera, S., Reker, D., Rigler, S. and Duncan, P. W. (2006). Therapeutic exercise and depressive symptoms after stroke. Fournal of the American Geriatrics Society, 54, 240-247.

Lord, S. E., Rochester, L., Weatherall, M., McPherson, K. M. and McNaughton, H. K. (2006). The effect of environment and task on gait parameters after stroke: a randomized comparison of measurement conditions. Archives of Physical Medicine and Rehabilitation, 87, 967-973.

Marzolini, S., Oh, P., McIlroy, W. and Brooks, D. (2013). The effects of an aerobic and resistance exercise training program on cognition following stroke. Neurorehabilitation and Neural Repair, 27, 392-402.

Meltzoff, A., Kuhl, P., Movellan, J. and Sejnowski, T. (2009). Foundations for a new science of learning. Science, $325,284-288$.

Nosek, B. A. and Banaji, M. R. (2001). The Go/No-Go association task. Social Cognition, 19, 625-666.

Penninx, B. W. et al. (2001). Physical exercise and the prevention of disability in activities of daily living in older persons with osteoarthritis. Archives of Internal Medicine, 161, 2309-2316. 
Peters, M., Chisholm, P. and Laeng, B. (1995). Spatial ability, student gender and academic performance. fournal of Engineering Education, 84, 60-73.

Potempa, K., Lopez, M., Braun, L. T., Szidon, J. P., Fogg, L. and Tincknell, T. (1995). Physiological outcomes of aerobic exercise training in hemiparetic stroke patients. Stroke; a fournal of Cerebral Circulation, 26, 101-105.

Pyöriä, O., Talvitie, U., Nyrkkö, H., Kautiainen, H., Pohjolainen, T., and Kasper, V. (2007). The effect of two physiotherapy approaches on physical and cognitive functions and independent coping at home in stroke rehabilitation. A preliminary follow-up study. Disability and rehabilitation, 29, 503-511.

Quaney, B. M. et al. (2009). Aerobic exercise improves cognition and motor function poststroke. Neurorehabilitation and Neural Repair, 23, 879-885.

Roth, D. L. (1989). Acute emotional and psychophysiological effects of aerobic exercise. Psychophysiology, 26, 593-602.

Shatil, S., Ivanova, T. D., Mochizuki, G. and Garland, S. J. (2005). Effects of therapeutic golf rehabilitation on golf performance, balance, and quality of life in individuals following stroke: pilot study. Physiotherapy Canada, 57, 101-112.

Shepherd, R. B. (2001). Exercise and training to optimize functional motor performance in stroke: driving neural reorganization? Neural Plasticity, 8, 121-129.
Strawbridge, W. J., Deleger, S., Roberts, R. E. and Kaplan, G. A. (2002). Physical activity reduces the risk of subsequent depression for older adults. American fournal of Epidemiology, 156, 328-334.

Truelsen, T., Piechowski-Jozwiak, B., Bonita, R., Mathers, C., Bogousslavsky, J. and Boysen, G. (2006). Stroke incidence and prevalence in Europe: a review of available data. European fournal of Neurology, 13, 581-598.

van de Port, I. G. L., Wevers, L. E. G., Lindeman, E. and Kwakkel, G. (2012). Effects of circuit training as alternative to usual physiotherapy after stroke: randomised controlled trial. BMF (Clinical Research ed.), 344, e2672.

Wevers, L., van de Port, I., Vermue, M., Mead, G. and Kwakkel, G. (2009). Effects of task-oriented circuit class training on walking competency after stroke: a systematic review. Stroke; a fournal of Cerebral Circulation, 40, 2450-2459.

Wiedenbauer, G. and Jansen-Osmann, P. (2007). Mental rotation ability of children with spina bifida: what influence does manual rotation training have? Developmental Neuropsychology, 32, 809-824.

Wohlschlaeger, A. and Wohlschlaeger, A. (1998). Mental and manual rotation. Fournal of Experimental Psychology. Human Perception and Performance, 24, 397-412.

Yang, Y.-R., Chen, Y.-C., Lee, C.-S., Cheng, S.-J., and Wang, R.-Y. (2007). Dual-task-related gait changes in individuals with stroke. Gait and Posture, 25, 185-190. 